\title{
COMPARISON OF FLUX PINNING IN SINGLE CRYSTALS AND MELT-TEXTURED $\mathrm{YBa}_{2} \mathrm{Cu}_{3} \mathrm{O}_{7-x}$
}

\author{
R. PUŹNiaK ${ }^{1}, A$. WIŚNIEWSKi ${ }^{1}$, M. Baran ${ }^{1}$ AND L. ZHoU ${ }^{2}$ \\ ${ }^{1}$ Institute of Physics, Polish Academy of Sciences \\ Al. Lotników 32/46, 02-668 Warsaw, Poland \\ ${ }^{2}$ Northwest Institute for Nonferrous Metal Research, Xi'an, P.R. China
}

Flux pinning properties of single crystals and melt-textured samples of $\mathrm{YBa}_{2} \mathrm{Cu}_{3} \mathrm{O}_{7-x}$ were studied for external magnetic field oriented parallel and perpendicular to the $\mathrm{CuO}_{2}$ planes ( $a b$-planes). For $H \| a b$ vortex behaviour is mainly determined by a very effective intrinsic pinning mechanism. The irreversibility line is located in the region of higher fields and temperatures in comparison with the irreversibility line for $H \perp a b$. In a single crystal for $H \perp a b$, we observed an increase in the critical current density $j_{\mathrm{c}}$ with magnetic field - the so-called "fishtail effect" - in a very broad temperature range. For $H \| a b$, this effect is observed only at temperatures close to $T_{c}$, when the intrinsic pinning is much weaker.

PACS numbers: 74.60.Ge, 74.60.Jg, 74.72.Bk

\section{Introduction}

Members of the homologous series of bismuth, thallium, and mercury based superconductors with a number of $\mathrm{CuO}_{2}$ planes $n>1$ have $T_{\mathrm{c}}$ s higher than $T_{\mathrm{c}}$ of $\mathrm{YBa}_{2} \mathrm{Cu}_{3} \mathrm{O}_{7-x}$ (Y123). However, anisotropy of $\mathrm{Y} 123(\gamma \approx 7)$ is much lower than these reported for Tl- or Hg-based compounds $(\gamma \approx 40$ for $n=3)$ and Bi-based compounds $(\gamma>150)$ [1]. Hence, at the liquid nitrogen temperature Y123 is still considered as the best material for large scale applications of HTSC. Flux pinning properties of HTSC differ strongly for external magnetic field oriented parallel and perpendicular to the $\mathrm{CuO}_{2}$ planes (ab-planes). For $H \| a b$, vortex behaviour is mainly determined by a very effective intrinsic pinning mechanism. For $H \perp a b$, i.e., $H \| c$-axis, coupling between quasi two-dimensional vortices located in adjacent sets of $\mathrm{CuO}_{2}$ layers is essential. In the paper we compare flux pinning properties of single crystals and melt-textured samples of $\mathrm{YBa}_{2} \mathrm{Cu}_{3} \mathrm{O}_{7-x}$ for both external magnetic field orientations. We have paid a special attention to the position of the irreversibility line (IL). Above this line, pinning vanishes due to thermally activated depinning. Hence, the position of the IL may be considered as a measure for the stability of pinning against thermal activation. 


\section{Experimental}

A single crystal was grown by the pulling method. The as-grown crystal was heated up to $800^{\circ} \mathrm{C}$ in oxygen atmosphere, then cooled down to $350^{\circ} \mathrm{C}$ and held for 3 weeks in order to get it fully oxygenated, as described in Ref. [2]. Magnetic susceptibility measurements performed on the post-annealed crystal along all principal axis directions indicated a sharp transition at $T_{\mathrm{c}}=87.4 \mathrm{~K}$. Melt-textured samples were prepared by the powder melting process [3]. The oxygen content was adjusted by an annealing treatment followed by quenching to room temperature. Measurements of $M(H)$ dependence were performed using a commercial Quantum Design SQUID magnetometer. The studied crystal was fully oxygenated hence, overdoped (oxygen deficiency $x=0 \pm 0.01$ ). One of the melt-textured samples was optimally doped $\left(T_{\mathrm{c}}=92.4 \mathrm{~K}, x=0.03\right)$, second slightly underdoped $\left(T_{\mathrm{c}}=89.4 \mathrm{~K}, x=0.16\right)$. A very small value of the field cooled magnetisation observed both for the single crystal and melt-textured samples indicate a rather strong flux pinning in the studied samples. In the case of a single crystal, mainly twin boundaries and crucible impurities act as strong pinning centres. In the case of melt-textured samples, the inclusions of $\mathrm{Y}_{2} \mathrm{BaCuO}_{5}$ (Y211) phase, with relatively big sizes (up to 1 micron) are mainly responsible for pinning. The irreversibility field, $H_{\text {irr }}$, was determined as a field of the appearance of the first resolved difference between lower and upper branches of the hysteresis loop.

\section{Results and discussion}

The temperature dependence of the irreversibility field for the Y123 single crystal and the melt-textured samples with $T_{\mathrm{c}}=92.4$ and $89.4 \mathrm{~K}$ for both magnetic field orientations is presented in Fig. 1. All the irreversibility lines are well described by a power law dependence $H_{\text {irr }}(T)=H_{\text {irr }}(0)\left(1-T / T_{\mathrm{c}}\right)^{\alpha}$. In the case of the single crystal we found $\alpha \approx 1.8$ and 1.6 , for $H \| a b$ and $H \| c$-axis, respectively. In the case of the melt-textured sample with $T_{\mathrm{c}}=92.4 \mathrm{~K}$ the exponent $\alpha \approx 1.6$ and 1.5 for $H \| a b$ and $H \| c$-axis, respectively. For the the melt-textured sample with $T_{\mathrm{c}}=89.4 \mathrm{~K}$ the respective values are $\alpha \approx 1.8$ and 1.5 . These values are quite

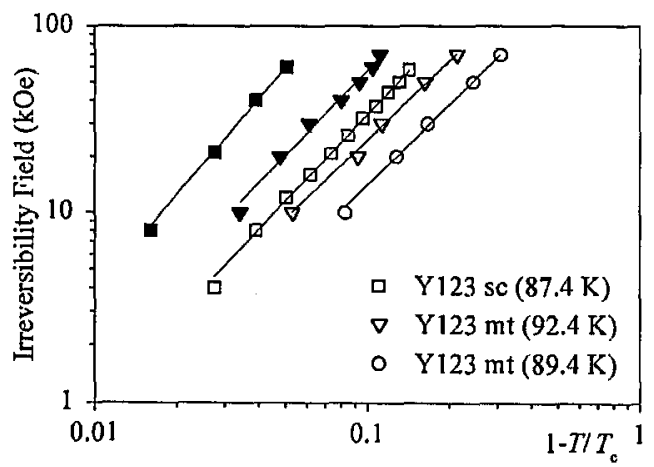

Fig. 1. Comparison of the irreversibility lines of the $\mathrm{YBa}_{2} \mathrm{Cu}_{3} \mathrm{O}_{7-x}$ single crystal and two melt-textured samples with $T_{c}=92.4$ and $89.4 \mathrm{~K}$. Open symbols denote data obtained for $H \| c$-axis and full symbols for $H \| a b$ orientation. 
close to the value $\alpha=2$ predicted for conventional melting of three-dimensional vortex lattice. As one can see the ILs for $H \| a b$ are always located in the region of higher fields and temperatures than the ILs for $H \| c$-axis (this is also the case for the IL of the melt-textured sample with $T_{\mathrm{c}}=89.4 \mathrm{~K}$ for $H \| a b$, which is not shown in Fig. 1 for clarity, since it overlaps with the IL of the single crystal for $H \| c$-axis). This results from a very effective intrinsic pinning, which confines the flux lines in between the $\mathrm{CuO}_{2}$ layers. The flux lines may only move parallel to the $a b$-plane and a large number of flux lines may be blocked even by a single defect if its pinning force is strong enough. The anisotropy of Y123, as well as of other HTSC, depends on doping level. Overdoped samples - in our case the fully oxygeneted single crystal - exhibit the smallest anisotropy, underdoped samples - in our studies the melt-textured sample with $T_{\mathrm{c}}=89.4 \mathrm{~K}$ - exhibit the highest anisotropy. Comparison of the position of the IL lines for $H \| c$-axis shows that in spite of different nature of the defects in the single crystal and melt-textured samples the position of the IL is determined by the anisotropy. The IL of the single crystal is located in the region of fields and temperatures significantly higher than the IL of the melt-textured sample with $T_{\mathrm{c}}=89.4 \mathrm{~K}$.
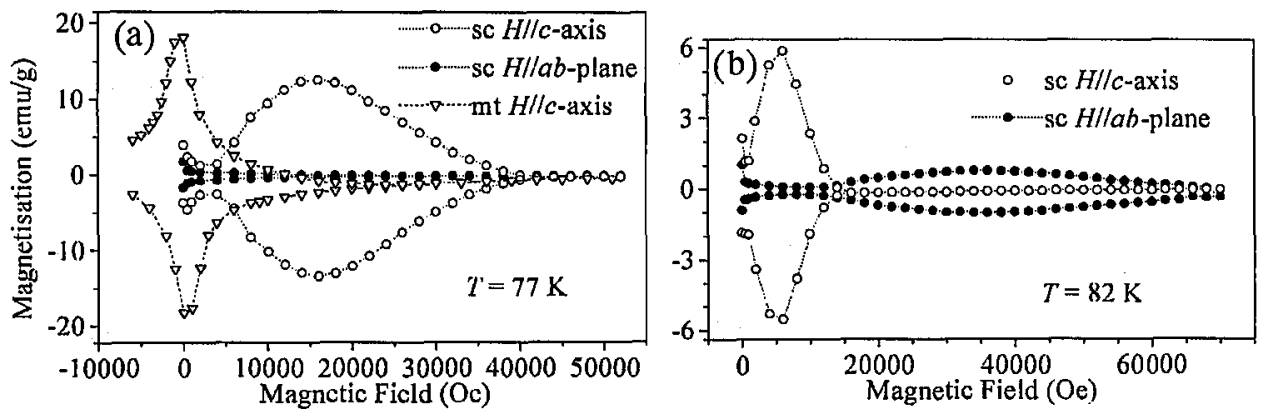

Fig. 2. (a) Comparison of the $M(H)$ loops at $77 \mathrm{~K}$ for the $\mathrm{YBa}_{2} \mathrm{Cu}_{3} \mathrm{O}_{7-x}$ single crystal and melt-textured sample with $T_{c}=92.4 \mathrm{~K}$ ( $H \| c$-axis). The $M(H)$ loop for $H \|$ $a b$ is not closed even at $50 \mathrm{kOe}$, which is not visible due to the scale of the $y$-axis. (b) Comparison of the $\mathrm{M}(\mathrm{H})$ loops at $82 \mathrm{~K}$ for the $\mathrm{YBa}_{2} \mathrm{Cu}_{3} \mathrm{O}_{7-x}$ single crystal.

In Fig. 2a comparison of the $M(H)$ hysteresis loops at $77 \mathrm{~K}$ for the single crystal ( $H \| c$-axis and $H \| \boldsymbol{a b}$ ) and for the melt-textured sample with $T_{\mathrm{c}}=92.4 \mathrm{~K}$ ( $H \| c$-axis) is shown. As one can see for $H \| c$-axis, at low fields $(<5 \mathrm{kOe})$, the width of $M(H)$ loop of the melt-textured sample is apparently bigger than that of the single crystal. Since the melt-textured sample and the single crystal are of the similar sizes the same is true for the critical current density $j_{\mathrm{c}}$. However, for $H \| c$-axis, the studied crystal exhibits a fishtail effect (a second maximum in the width of hysteresis loop) in the whole investigated temperature range (30-87 K). According to the Bean critical state model the consequence of the fishtail shape of a $M(H)$ loop is an increase in the critical current with increasing magnetic field. In fact, transport measurements [4] showed a good correspondence with magnetisation measurements. As one can see, this effect is very pronounced at $77 \mathrm{~K}$. In a field of about $16 \mathrm{kOe}$ (the maximum of the fishtail effect at $77 \mathrm{~K}$ ) 
the width of $M(H)$ loop is more than one order of magnitude bigger than that of the melt-textured sample. For $H \| a b$, this effect is observed only at $T>81 \mathrm{~K}$ (see Fig. 2b), i.e., very close to $T_{\mathrm{c}}$, when the intrinsic pinning is much weaker. We have not observed any fishtail effect for the melt-textured samples.

Since the studied single crystal is fully oxygenated, we can rather exclude the effect of the field-induced granularity due to oxygen deficient areas [5] as a reason of the fishtail phenomenon. We have also checked that at different temperatures the pinning force in the fishtail region scales with $h=H / H_{\text {irr }}$. This indicates that temperature independent geometric matching effects (e.g. matching of the vortex lattice with the twins structure) do not play an important role as a pinning mechanism. The studied crystal contains impurities due to crucible corrosion. The impurities can act as a strong pinning centres. The fishtail effect is observed in majority of HTSC single crystals. This indicates that the phenomenon is not a material specific property, but some general property of the flux line lattice (FLL). With increasing magnetic field elastic properties of the FLL change. The softening of the FLL, mainly due to the decrease in shear modulus, enables a better adjustment of the lattice to the defect structure existing in the crystal. As the consequence FLL becomes more stable against the Lorentz force until the optimal configuration for a given defect structure and temperature is reached, giving rise to the (second) maximum in $M(H)$ loop. When the field increases further the defects are unable to pin more vortices and the width of the loop (critical current) decreases. On the other hand, the lack of the fishtail effect in the melt-textured samples may be explained assuming that the flux pinning due to the Y211 inclusions is so strong that the softening of the FLL does not provide any possibility of the more effective flux pinning in higher magnetic fields. In the case of $H \| a b$ configuration, a very effective intrinsic pinning is responsible for the FLL behaviour. However, close to $T_{\mathrm{c}}$ the coherence length along the $c$-axis significantly increases and the effect of the intrinsic pinning becomes weak. Then the movement of the vortex along the $c$-axis is possible and the FLL may better adjust to the defects structure existing in the sample, which results in the $j_{\mathrm{c}}$ enhancement.

\section{Acknowledgment}

This work was partially supported by the Committee for Scientific Research under contract No. 2 P03B 09514.

\section{References}

[1] M. Thinkam, Introduction to Superconductivity, McGraw-Hill Inc., New York 1996, p. 321.

[2] S. Miyamoto, Y. High, J. Schutzmann, H. Kutami, Y. Yamada, S. Tajima, S. Shiohara, in: Advances in Superconductivity VII, Eds. K. Yamafuji, T. Morishita, Springer-Verlag, Tokyo 1995, p. 129.

[3] L. Zhou, P. Zhang, P. Ji, K. Wang, X. Wu, Supercond. Sci. Technol. 3, 490 (1990).

[4] S.N. Gordeev, W. Jahn, A.A. Zhukov, H. Kupfer, T. Wolf, Phys. Rev. B 49, 15420 (1994).

[5] M. Daeumling, J.M. Seutjens, D.C. Larbalestier, Nature 346, 332 (1990). 